\title{
Rethinking the role of the brain in glucose homeostasis and diabetes pathogenesis
}

\author{
Jenny M. Brown, ${ }^{1}$ Jarrad M. Scarlett, ${ }^{1,2}$ and Michael W. Schwartz ${ }^{1}$ \\ 'University of Washington Medicine Diabetes Institute, Department of Medicine, and ²Department of Pediatric Gastroenterology and Hepatology, University of Washington, Seattle, Washington, USA.
}

$\mathbf{T}_{1}$ he brain plays a major role in homeostatic processes ranging from control of body temperature and fat mass to blood pressure and volume. Tight regulation of the circulating glucose level is similarly crucial for survival, and since the brain relies almost exclusively on glucose as a fuel source, it seems counterintuitive to think that the brain does not also play an important role in glucose homeostasis. Based on overwhelming evidence supporting the endocrine pancreas as the primary controller of the blood glucose (BG) level, however, the notion of a key role for the brain was discounted decades ago.

Yet recent findings are beginning to chip away at the foundation of the prevailing, islet-centered view of glucose homeostasis. This perceptual shift is being driven not by evidence against a role for the endocrine pancreas in glucose homeostasis, but by evidence that the endocrine pancreas is part of a larger regulatory system, the activity of which is integrated with other critical homeostatic control systems governed by the brain.

\section{Integration of glucose \\ homeostasis with energy homeostasis and thermoregulation}

This countervailing narrative begins with recognition that the amount of insulin secreted in response to a glucose challenge can be dynamically regulated by both humoral and autonomic inputs. Pancreatic islets are richly innervated by both sympathetic and parasympathetic fibers, with the former capable of powerfully inhibiting glucose-stimulated insulin secretion (GSIS) and the latter having the opposite effect $(1,2)$. More importantly, growing evidence that physiologically important changes in both insulin secretion and tissue glucose utilization can occur in the absence of any change in the BG level suggests that pancreatic $\beta$ cell function can be regulated as part of a larger system for controlling glucose homeostasis.

For an illustration of this concept, consider that across much of the planet, mammals are confronted with swings in environmental temperature on a daily basis that pose a substantial homeostatic challenge. Meeting this challenge requires activation of diverse metabolic and autonomic responses involving three distinct homeostatic systems - glucose homeostasis, energy homeostasis, and thermoregulation - that must be integrated seamlessly if body temperature, body fat stores, and BG levels are to be maintained within narrow physiological limits.

In response to cold exposure, heat production must increase in a rapid and sustained manner if hypothermia is to be avoided, and the sympathetic nervous system (SNS) plays a key role in driving this process $(3,4)$. Specifically, activation of SNS outflow to thermogenic tissues (e.g., brown and white adipose tissue, skeletal muscle), driven by thermoregulatory neurocircuits situated in the hypothalamic preoptic area, increases heat production via a mechanism that is highly reliant on oxidation of glucose as a substrate $(3,4)$. Beyond preserving core temperature, two additional challenges thus confront the cold-exposed animal: (a) how to preserve energy balance in the face of markedly increased rates of energy expenditure and (b) how to preserve stable glycemia in the face of markedly increased rates of glucose utilization.

As a result of integration across these three regulatory systems, cold exposure increases food intake in a manner that somehow - precisely offsets the increase

Conflict of interest: Funding in support of this work was provided to MWS by Novo Nordisk A/S (CMS-431104). Copyright: (5) 2019, American Society for Clinical Investigation.

Reference information: J Clin Invest. 2019;129(8):3035-3037. https://doi.org/10.1172/JCI130904. 
A Ambient temperature

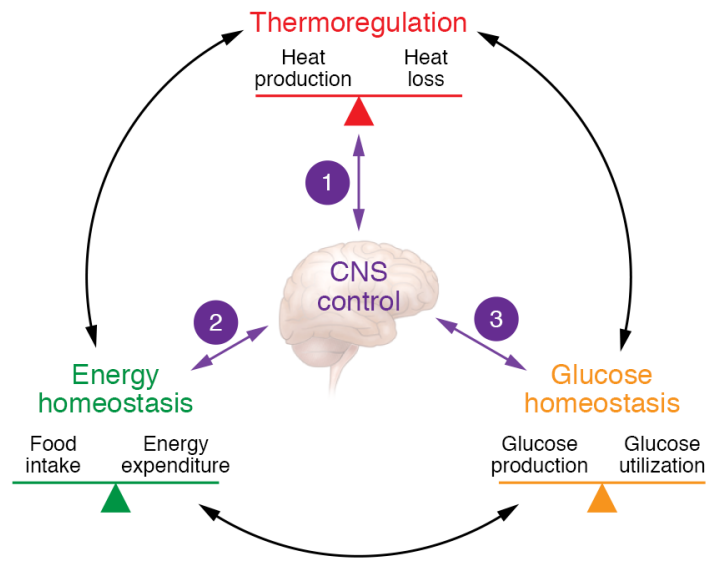

B Cold temperature exposure

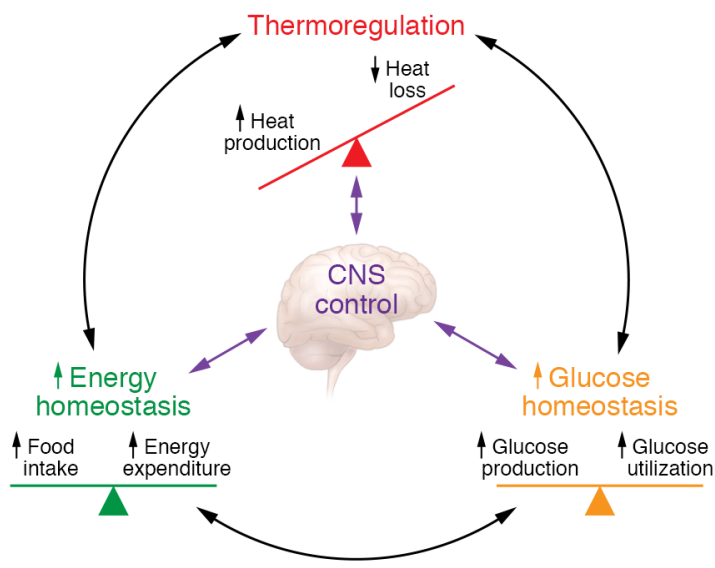

Figure 1. Model for integrated central control of body temperature, fat mass, and blood glucose levels. (A) Maintenance of body temperature, body fat stores, and blood glucose levels within narrow physiological limits requires seamless integration of systems governing thermoregulation, energy homeostasis, and glucose homeostasis. This integration is coordinated by the brain, and it is dependent upon accurate sensing by the brain of external temperature (1), body fat content (2), and the blood glucose level (3). (B) During cold exposure, the increased demand for heat production is met through markedly increased rates of glucose utilization by thermogenic tissues. Energy homeostasis is preserved by a centrally mediated increase in food intake, while glucose homeostasis preserved by centrally mediated inhibition of insulin secretion (to avert hypoglycemia). Impaired sensing of the relevant afferent input results in a compensatory increase in the defended level of the regulated variable. In the case of T2D, impaired brain glucose sensing is hypothesized to raise the defended blood glucose level into the diabetic range, with inhibition of insulin secretion playing a key role.

is that although there is an increase in the level of the regulated variable - blood glucose, blood pressure, or body fat mass - the underlying homeostatic control mechanisms appear to function normally. Since these three disorders cluster together as part of the metabolic syndrome, the possibility of a shared regulatory defect can be considered.

What mechanisms drive the defense of hyperglycemia in T2D? While $\beta$ cell dysfunction clearly plays a role, an important unanswered question is whether this reflects a $\beta$ cell-autonomous defect or is instead imposed upon $\beta$ cells by the brain (analogous to the reduction in GSIS observed during cold exposure). Consistent with the latter notion is that sympathetic inhibition of insulin secretion is increased in patients with T2D (9).

Studies in mice have identified a distinct subset of neurons in the hypothalamic ventromedial nucleus (VMN) that, when activated, not only induce diabetes-range hyperglycemia, but also completely block GSIS (10). If the brain were to perceive the BG level to be lower than it truly is, it conceivably could mount responses (including GSIS inhibition) that raise the defended level of glycemia. Indeed, the response to experimentally induced neuroglucopenia establishes this to be the case. Neuroglu- copenia is induced by administration of a nonmetabolizable glucose analog (e.g., 2-deoxy-D-glucose), which is transported into cells but cannot be metabolized further, thereby disrupting cellular glucose metabolism. In response, the brain rapidly raises the BG level, which serves as a readout for whether neuroglucopenia was in fact achieved (11). Moreover, the aforementioned VMN neurons are implicated as drivers of this hyperglycemic response (12). These observations collectively support a model whereby defective brain glucose sensing contributes to the pathogenesis of hyperglycemia in $\mathrm{T} 2 \mathrm{D}$, analogous to the effect of impaired leptin sensing in driving excessive accumulation of body fat.

The progressive nature of $\beta$ cell dysfunction in $\mathrm{T} 2 \mathrm{D}$, culminating in overt $\beta$ cell failure, would at first glance seem to challenge this model of disease pathogenesis, since it is not immediately clear how this progression might result from a defect that does not reside within the $\beta$ cell itself Despite a decades-long search, however, a cell-autonomous basis for progressive $\beta$ cell failure remains to be identified. Moreover, most endocrine cell types become severely atrophic and dysfunctional if they are subjected to continuous inhibition over long time intervals. Investigation into the contribution to $\beta$ cell dysfunction made by tonic inhibition arising from the brain, perhaps aggravated by worsening metabolic status (e.g., hyperglycemia and associated glucose toxicity) and/or genetic susceptibility, is a key priority for future study.

Does T2D pathogenesis involve aberrant activity of hypothalamic glucoregulatory neurocircuits, and is this capable of raising the defended level of glycemia? Although our understanding of glucoregulatory neurocircuitry is in its infancy, available evidence indicates that (i) fuelsensing neurocircuits are concentrated in the mediobasal hypothalamus ( $\mathrm{MBH})$ and (ii) some of these circuits are overactive in rodent models of diabetes. Among these are GABAergic neurons situated in the arcuate nucleus that express both agoutirelated peptide (Agrp) and neuropeptide Y (NPY) (referred to as Agrp neurons) (7). These neurons are physiologically important regulators of both food intake and glycemia, and they are tonically inhibited by humoral signals that convey information regarding the status of either stored fuel (e.g., leptin) or fuel available for immediate use (glucose) $(13,14)$. Consequently, these neurons are activated by low plasma levels of either leptin or glucose, and in otherwise normal mice, this activation is sufficient to both stimulate food intake and elevate the $B G$ level into the diabetic range, while 
conversely, silencing of these neurons is sufficient to ameliorate hyperglycemia in diabetic $d b / d b$ mice (13). That these neurons are activated across rodent models of diabetes (15-17) makes them an attractive candidate mediator of the defense of hyperglycemia in T2D. The contribution made by other glucoregulatory neurons (e.g., in the $\mathrm{VMN}$ ) to diabetic hyperglycemia is under active investigation.

\section{Therapeutic implications}

Since Agrp neurons are activated by hypoglycemia/neuroglucopenia (18), it seems paradoxical that they should also be activated in diabetic, hyperglycemic animals, and yet this is clearly the case (15-17). To explain this paradox, we hypothesize that brain sensing of glucose and other fuels is impaired in T2D and that hypothalamic glucoregulatory neurocircuits are activated as part of a compensatory response that drives an increase in BG level (in part by inhibiting GSIS). This model of T2D pathogenesis predicts that correcting the underlying defect should normalize glycemia in diabetic animals. Notable in this regard is the sustained antidiabetic action induced by central administration of FGF1 (19-22). In rodent models of T2D, remission of hyperglycemia can be sustained for weeks or months following a single intracerebroventricular injection of FGF1. The underlying mechanism remains under active study, but instead of simply lowering the BG level, FGF1 appears to act on MBH neurocircuits to reset glycemia in the normal range. Such an effect would not seem possible unless (a) the brain plays a key role in establishing the BG level and (b) a defect in this system contributes to the pathogenesis of hyperglycemia in these animal models.

\section{Conclusion}

The notion that glucose homeostasis is governed primarily by the pancreas, rather than the brain, has come under increasing scrutiny in the wake of findings that simply cannot be explained by this model. Particularly noteworthy is evidence that in rodent models of $\mathrm{T} 2 \mathrm{D}, \mathrm{BG}$ can be restored to normal for weeks or months by targeting of brain systems controlling glucose homeostasis. Fortunately, recent advances in neuroscience offer an unprecedented ability to map and functionally characterize the relevant neurocircuits in rodent models (7). We should seize upon this opportunity to advance our understanding of how glucose homeostasis is regulated by the brain, identify the contribution made by defects in this regulatory system to the pathogenesis of $\mathrm{T} 2 \mathrm{D}$, determine whether such findings substantially translate to humans, and if so, investigate whether these insights offer novel approaches to more effective disease treatment.

\section{Acknowledgments}

This work was supported by National Institute of Diabetes and Digestive and Kidney Diseases grants DK101997, DK083042, and DK035816 (to MWS) and DK114474 (to JMS). JMB is supported by National Heart, Lung, and Blood Institute T32 Training Grant HL007312 and by the University of Washington Diabetes Research Center Samuel and Althea Stroum Endowed Graduate Fellowship.

Address correspondence to: Michael W. Schwartz, Department of Medicine, University of Washington at South Lake Union, 850 Republican St, N335, Box 358055, Seattle, Washington 98195, USA. Phone: 206.897.5288; Email: mschwart@u.washington.edu.

1. Rosario W, et al. The brain-to-pancreatic islet neuronal map reveals differential glucose regulation from distinct hypothalamic regions. Diabetes. 2016;65(9):2711-2723.

2. Thorens B. Central control of glucose homeostasis: the brain-endocrine pancreas axis. Diabetes Metab. 2010;36(suppl 3):S45-S49.

3. Tan CL, Knight ZA. Regulation of body temperature by the nervous system. Neuron. 2018;98(1):31-48.

4. Morrison SF, Madden CJ, Tupone D. Central neural regulation of brown adipose tissue thermogenesis and energy expenditure. Cell Metab. 2014;19(5):741-756.

5. Kaiyala KJ, Ogimoto K, Nelson JT, Schwartz MW, Morton GJ. Leptin signaling is required for adaptive changes in food intake, but not energy expenditure, in response to different thermal conditions. PLoS One. 2015;10(3):e0119391.

6. Morton GJ, et al. Evidence that the sympathetic nervous system elicits rapid, coordinated, and reciprocal adjustments of insulin secretion and insulin sensitivity during cold exposure. Diabetes. 2017;66(4):823-834

7. Lowell BB. New neuroscience of homeostasis and drives for food, water, and salt. N Engl JMed. 2019;380(5):459-471.

8. Holman RR, Turner RC. Maintenance of basal plasma glucose and insulin concentrations in maturity-onset diabetes. Diabetes. 1979;28(3):227-230.

9. Robertson RP, Halter JB, Porte D. A role for $\alpha$-adrenergic receptors in abnormal insulin secretion in diabetes mellitus. J Clin Invest. 1976;57(3):791-795.

10. Faber CL, et al. Distinct neuronal projections from the hypothalamic ventromedial nucleus mediate glycemic and behavioral effects. Diabetes. 2018;67(12):2518-2529.

11. Molina PE, et al. Hormonal and metabolic effects of neuroglucopenia. Brain Res. 1993;614(1-2):99-108.

12. Meek TH, et al. Functional identification of a neurocircuit regulating blood glucose. Proc Natl Acad Sci U S A . 2016;113(14):E2073-E2082.

13. $\mathrm{Xu}$ J, et al. Genetic identification of leptin neural circuits in energy and glucose homeostases. Nature. 2018;556(7702):505-509.

14. Beutler LR, Chen Y, Ahn JS, Lin YC, Essner RA, Knight ZA. Dynamics of gut-brain communication underlying hunger. Neuron. 2017;96(2):461-475.e5.

15. Schwartz MW, et al. Specificity of leptin action on elevated blood glucose levels and hypothalamic neuropeptide Y gene expression in ob/ob mice. Diabetes. 1996;45(4):531-535.

16. Havel PJ, et al. Effects of streptozotocininduced diabetes and insulin treatment on the hypothalamic melanocortin system and muscle uncoupling protein 3 expression in rats. Diabetes. 2000;49(2):244-252.

17. Park ES, et al. Changes in orexin-A and neuropeptide y expression in the hypothalamus of obese and lean Zucker diabetic fatty rats. J Vet Med Sci. 2005;67(7):639-646.

18. Han S, Chen X, Wu YM, Naes L, Westfall T. Elevated neuropeptide $\mathrm{Y}$ gene expression and release during hypoglycemic stress. Peptides. 1997;18(9):1335-1340.

19. Scarlett JM, et al. Central injection of fibroblast growth factor 1 induces sustained remission of diabetic hyperglycemia in rodents. Nat Med. 2016;22(7):800-806.

20. Scarlett JM, et al. Peripheral mechanisms mediating the sustained anti-diabetic action of FGF1 in the brain. Diabetes. 2019;68(3):654-664.

21. Brown JM, et al. The hypothalamic arcuate nucleus-median eminence is a target for sustained diabetes remission induced by fibroblast growth factor 1. Diabetes. 2019;68(5):1054-1061.

22. Tennant KG, Lindsley SR, Kirigiti MA, True C, Kievit P. Central and peripheral administration of fibroblast growth factor 1 improves pancreatic islet insulin secretion in diabetic mouse models. Diabetes. 2019;68(7):1462-1472. 